\title{
Antisymmetric Relationship
}

National Cancer Institute

\section{Source}

National Cancer Institute. Antisymmetric Relationship. NCI Thesaurus. Code C75920.

A binary relation such that if $a$ is related to $b$ and $b$ is related to $a$, then $a$ equals $b$. 